\title{
A New Microscopy Facility for Optical, Electron and X-Ray Imaging at Diamond
}

Paul Quinn ${ }^{1}$, Julia Parker ${ }^{1}$ and Fernando Cach-Nerin ${ }^{1}$, Dogan Ozakaya ${ }^{2}$, Judy Kim ${ }^{1,3}$ and Angus Kirkland $^{1,3}$

1. Diamond Light Source Ltd., Harwell Science and Innovation Campus, Didcot, UK.

2. Johnson Matthey Technology Centre, Sonning Common, Reading, Reading, UK

3. Department of Materials, Parks Road, Oxford, UK.

Multi-scale, multi-technique experiments combining optical, X-ray and electron microscopy enable studies of systems and processes at different levels of complexity. This broader view is obtained from the differences in resolution and more importantly when the physical properties of the probes such as penetration depth or contrast mechanisms are exploited to view the sample or process in a different light.

In the life sciences fluorescence microscopy is increasingly used to identify areas and events of interest within cells which are then examined at high resolution by electron microscopy. More recently these techniques have been combined with soft X-ray cryo transmission microscopy to bridge the length scales between these techniques to provide a more complex, complimentary view of a full (nonsectioned) cell [1]. In the physical sciences a recent study of nanocatalysts in operando conditions which correlated scanning transmission electron microscopy and X-ray spectroscopy clearly demonstrates the potential of an integrated approach [2].

The advantages of combining these various techniques are clear but they require substantial resources for equipment, infrastructure and expertise. To exploit the latest cutting edge developments in electron microscopy and to facilitate correlation of these techniques a new facility housing the I14 beamline and a National Electron Microscopy Facility for Physical Sciences (EMPSF) will open in late 2016 at Diamond Light Source. Within the EMPSF, Johnson Matthey, the University of Oxford and Diamond Light Source are collaborating on the creation of a state-of-the-art materials characterization facility. The core builiding will also house a National Facility for Cryo-Electron Microscopy - the electron BioImaging Centre (eBIC) - with two advanced electron microscopes funded by The Wellcome Trust, MRC and BBSRC, in an initiative led by Birkbeck College, London and the University of Oxford [3].

The $\mathrm{I} 14$ beamline is a $185 \mathrm{~m}$ long facility for hard X-ray scanning probe microscopy which will operate over a $4.5-26 \mathrm{keV}$ range and deliver an initial probe size of $30 \mathrm{~nm}$. The experimental station is designed for scanning X-ray fluorescence, X-ray spectroscopy and diffraction and features a cryo-sample mount with rotation for cryo-tomography.

eBIC will provide two state-of-the-art $300 \mathrm{kV}$ FEI Titan-Krios cryo-microscopes with stable, cartridgetype sample holders and the latest generation of electron detectors, incorporating correction for beaminduced motion, as well as phase plates. The demand for these facilities is such that the first of these instruments was installed on the synchrotron hall floor in April 2015 and has been operating a full user program since startup. The second instrument is to be installed within the synchrotron in early 2016 with both devices subsequently migrating to the new building.

Oxford University will bring a unique JEOL $300 \mathrm{kV}$ electron microscope dedicated to atomic scale imaging at world-leading resolution over a range of primary voltages and Johnson Matthey will install a 
JEOL double-EDX and EELS capable microscope dedicated to chemical analysis at atomic scale resolution. The co-location of these instruments at a synchrotron facility - in particular with I14 - will provide exciting scientific opportunities for materials science. Collaborations between Johnson Matthey, Oxford University and the I14 beamline are underway to facilitate the interchange of samples and sample environments between these instruments by developing an exchangeable sample holder which should enable analyses at near in-operando catalytic conditions across the instruments. Of particular interest is the development of tomography for the observation of porosity and 3D chemical analysis. In addition to the hardware developments there is considerable overlap with beamline data analysis, image and tomography processing and data curation which is leading to collaborations and developments which will benefit both communities.

The organization and operation of the electron microscopy facilities is modelled on the Diamond beamlines. The core user program will be based on the six-month Diamond beamtime application system with peer-reviewed access via an independent panel. Diamond beamlines typically offer around 200 days of 24-hr user X-ray beam time per year and the goal is for the eBIC facility to match this with the materials characterization EM facility offering roughly $50 \%$ of this for users. The increased in-house allocation for material science will be a key factor to developing sample exchange systems.

These facilities are supported by labs equipped for advanced sample preparation for materials science and life science with plasma cleaning, high-pressure freezing, cryo-sectioning and a dual-beam cryoSEM complimented by the wide range of instrumentation and preparation tools already available in the synchrotron beamline support facilities, such as cryo-fluorescence microscopes, IR and Raman microscopes.

This concentration of equipment and expertise within this facility should provide new exciting opportunities for multi-modal high resolution scientific studies.

\section{References:}

[1] Zeev-Ben-Mordehai, T., Hagen, C. \& Grunewald, K. Virology 5 (2014), 42

[2] Y Li, et al Nature Communications 6 (2015), 7583

[3] Saibil, H. R., Grunewald, K. \& Stuart, D. I Acta Cryst. D 71, (2015), 127

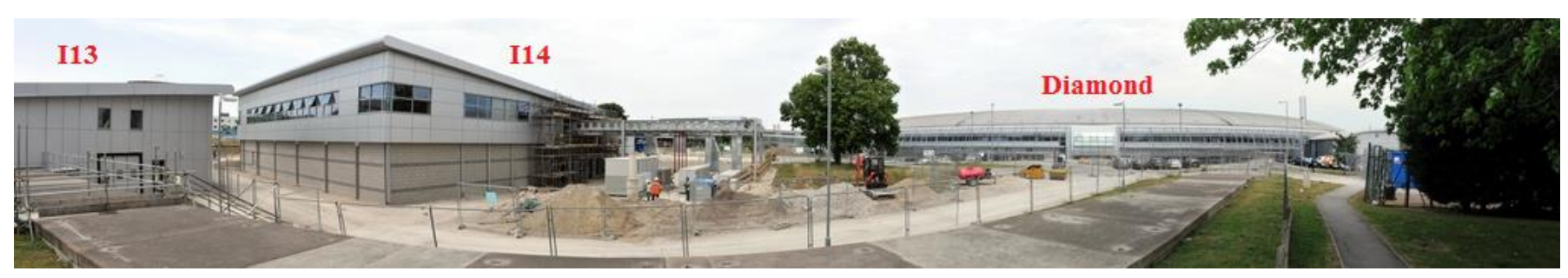

Figure 1. Panoramic view of the I14 and electron microscopy building under construction at Diamond. 